\title{
Design Of Adjustable Air Suction Seeder With Plug Board
}

\author{
Lin $\mathrm{He}^{1, \mathrm{a}}$, Xiao-rong LÜ1, ${ }^{*}$, Xiao Wang ${ }^{1, \mathrm{c}}$,Teng-fei $\mathrm{Wu}^{1, \mathrm{~d}}$ \\ 1 Sichuan Agricultural University, 46 xinkang road, Ya'an ,Sichuan, China \\ a572942740@qq.com, ${ }^{* b} \mid x r x j 2008 @ 163 . c o m,{ }^{c 934940773 @ q q . c o m,{ }^{d} 1484671243 @ q q . c o m}$
}

\section{Keywords: Sowing device,Air suction,Peanuts sowing,Board}

Abstract. In order to improve the production efficiency of peanuts in China and increase the yield,a kind of adjustable air suction seeder with plug board is designed for peanut seeding . The seeder is designed for efficient seeding of large seeds such as peanuts. This structure can quickly finish the replacement of the top block during the machine sowing process, and then to achieve the adjustments of seeding rate and seeding spacing of different crops.

\section{Introduction}

Peanut is an important oil crop and high quality protein resources in China,and it is also an important export of foreign exchange products and agricultural restructuring to focus on the development and support direction ${ }^{1}$. China is not only the major peanut producer and peanut consumer of the world, but also the largest peanut exporter.Sowing is an important part of peanut production process.Liberation of peanut mechanization can not only increase the productivity of labor, but also to improve production efficiency and promote increased production and income.As the air suction seeding device on the seed size is not strict, do not need to select the classification, less injury, easy to achieve a single grain on demand, planer to improve the speed of operation and so on, has been widely studied and applied ${ }^{2}$.The pitch adjustment of the existing air suction peanut seeding machine is generally done by replacing the sucking plate and the duckbill assembly with different number of suction holes. Since the sucking plate is installed inside the grouting wheel,disassembling to complete the replacement of the suction tray, so loading and unloading is very troublesome.In addition, the current air suction sowing machine can not change the number of planting per hole, which for the user there is a big trouble.In this paper, we design an adjustable air suction sowing device, which can change the movable baffle movement interval by rapidly changing the number of tops of different shapes or increasing the number of top blocks according to the planting pattern and the seeding number,so as to effectively achieve the seeding process during the planting of the number of seed and sowing pitch changes.

\section{The structure and working principle of adjustable air suction seeder with plug board}

Overall structural design. The adjustable air suction seeding device with plug board be disigned in the paper is composed of air chamber, movable partition, fixed partition, top block, cardboard, column, block cover, slip sleeve, compression spring, connecting plate and convex body.Figure 1, 2, and 3 shows the structure of the seeder. 


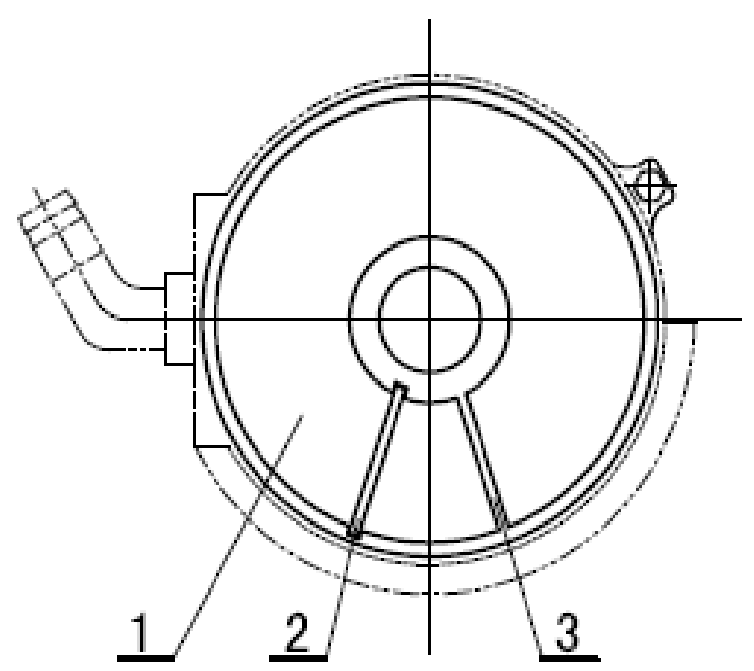

Fig.1 Schematic diagram of the gas chamber structure of the seeder 1,Air chamber 2,Active baffle 3,Fixed baffle 4,Top block

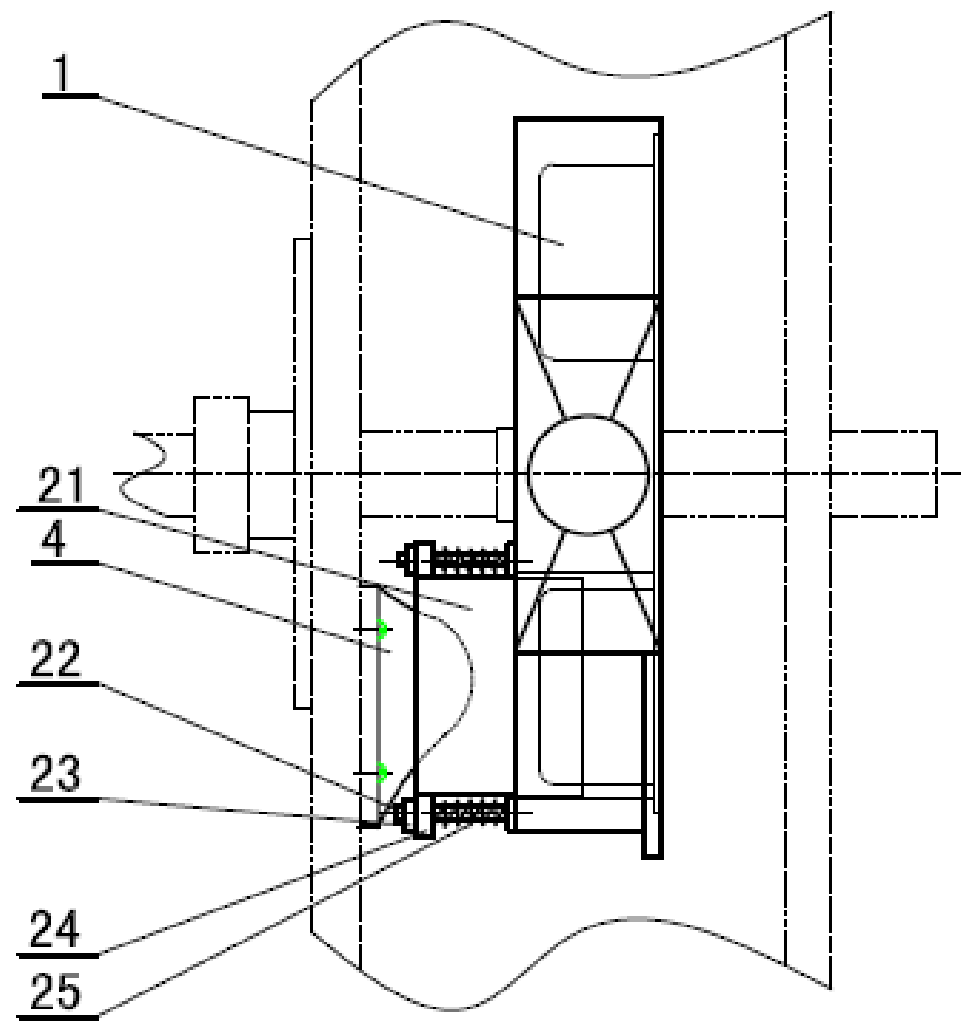

Fig.2 A schematic view of the side view of the seeder

1 Air chamber,4 Top block,21 Board,22 Column,23 Gear cover,24 Gear cover,25 Compression spring

working principle.Before seeding, according to the seeding pattern and the number of seeding requirements, in the seeder on the drum side of the installation of the corresponding number and shape of the top block, (When the speed of the drum and the speed of the suction tray are the same, the more the top block is installed on the same circumference of the drum side, the smaller the pitch of the seeding is, the longer the time when the top block is pushed into the air chamber ${ }^{3}$. that is, the longer the time to cut off the gas source, the more the number of particles per hole)in order to achieve the seeding process of seeding pitch and the number of seeds per hole adjustment. 
At the time of seeding, with the operation of the seeding machine, the machine pulls the hill drop wheel to turn, and drives the sucking plate relative to the seed chamber through the drive shaft. The air chamber rotates and the negative pressure is applied in the air chamber, which brought the seed out from seed chamber.When the suction tray is transferred to the lower portion of the chamber, the flap in the movable partition is moved along the inner wall of the chamber under the action of the top block on the rotating drum side panel to separate the air chamber into two cavities,and cut off the lower chamber air supply. The movable partition and the fixed partition divide the chamber into two chambers, the upper chamber is the vacuum zone, the lower chamber is the non-vacuum zone. When the sucking plate to the lower chamber non-vacuum area,due to suction negative pressure disappeared, the seeds rely on their own weight to fall, to achieve the sowing process. When the top block leaves the board, the board is pressed under the action of the compression spring, the chamber chamber to restore negative pressure. When the sucking tray passes through the area, the seed is allowed to pass through the negative pressure action.

\section{The design of the main working parts of the adjustable air suction seeder with plug board}

Design of movable partitions.As shown in Fig2,the movable partition consists of a card, a column, a canopy, a slip sleeve and a compression spring. The card is fixed with the sliding sleeve,the column is fastened to the outer wall of the air chamber, which is then fitted with a compression spring, a slip sleeve and a casing. The movable bulkhead can move along the inner wall of the air chamber. When the top block acts on the movable partition, the movable partition can enter the air chamber and together with the fixed partition in the air chamber to form two cavities, namely, the vacuum chamber and the non-vacuum chamber body ${ }^{4}$. When the top block leaves the card, the board can be reset under the action of a compression spring.

Design of top blocks.As shown in Fig. 3, the top block is composed of a connecting plate and a convex body,the connecting plate is connected with the side wall of the drum, and the upper body is convex, the convex body is trapezoidal, the inclination of the front side is small, which is easy to push the board slowly, and the rear side of the slope is convenient for the board to quickly reset.The longer the straight line on the plane, the longer the residence time of the board in the air chamber, the effective increase in the number of seeds per hole. The more the number of the top blocks are installed on the same circumference as the position of the corresponding side of the cylinder, the smaller the pitch of the seeding.

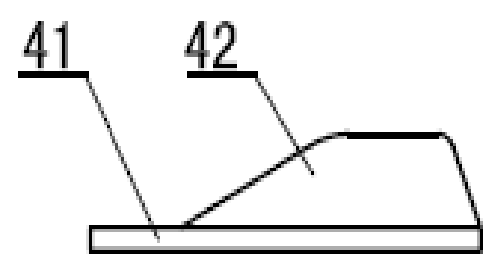

Fig.3 Schematic diagram of side view of seeder top block 41 Connection plate, 42 Convex body

\section{Features of Adjustable Sampler for Air Paddle}

The structure of the adjustable air suction sowing device is shown in Fig. 1 and Fig. 2, and the movable block is moved along the inner wall of the chamber. When the movable partition enters into the air chamber and forms two cavities with the fixed partition, namely, a vacuum chamber and a non-vacuum chamber.

The top block is trapezoidal, the front side of the slope is small, conducive to the board is slowly pushed to be difficult to be stuck, the rear side of the slope of the large use of rapid reset.The longer 
the upper plane of the top block, the longer the residence time of the board in the air chamber, effectively increasing the number of seeds per hole. The more the number of the top blocks are installed on the same circumference as the position of the corresponding position on the side of the drum, the smaller the pitch.Therefore, the length of the plane on the top block can affect the seeding rate, the design of the number of top blocks can affect the pitch.

\section{Conclusion}

The whole structure of the seeder is simple and novel, easy to operate, and can save time; for peanuts and other large seeds of precision seeding, can quickly increase and tear the number of top and bottom to achieve seeding adjustment, improve the quality of planting and the seeding efficiency.

The seeding device adopts the active baffle structure, and effectively achieves the adjustability of planting by the effective cooperation with the top block, thus effectively achieving the change of seeding number and seeding distance in each seeding process.

\section{Acknowledgement}

The study was supported by the Jiangsu Natural Science Foundation of China (BK2012518.)

Correspondence Author: Lv Xiaorong, College of Information \& Engineering, Sichuan Agricultural University, Ya'an, China, 1xrxj2008@163.com.

\section{References}

[1] Yang Mengfan. Peanut service rate increase need to increase support [N]. Grain Market News, 2017-06-03 (B01).

[2] SUN Yu-tao, TIAN Li-zhong, SHANG Shu-qi, YANG Sui-bing, WANG Yan-yao, ZHAO Jian-liang. Performance test of the inside of the peanut seeder on the inside of the seeder $[\mathrm{J}]$. Chinese Journal of Agricultural Engineering, 2012,28 (S2): 84-89. [2017-10-13].

[3] Ling Xuan, Wang Xudong. Peanut seeder inside filling seeder design and test [J]. Modern Agricultural Equipment, 2014, (05): 47-51. [2017-10-13].

[4] Zhou Liangyong. Series of new peanut seeder [J]. New Rural Technology, 2003, (02): 20. [2017-10-13]. 